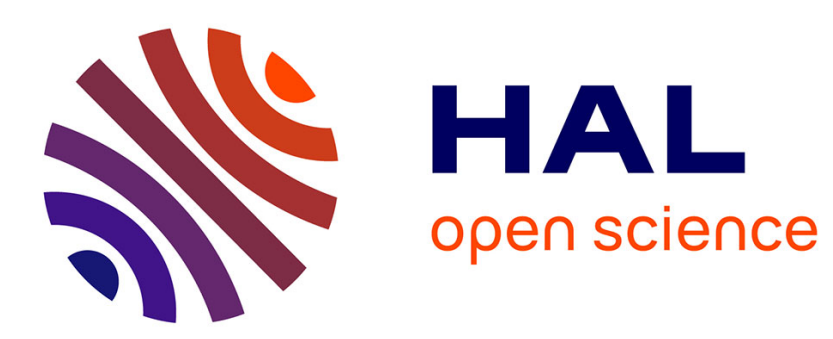

\title{
Estimation of fractional integration under temporal aggregation
}

\author{
Uwe Hassler
}

\section{To cite this version:}

Uwe Hassler. Estimation of fractional integration under temporal aggregation. Econometrics, 2011, 10.1016/j.jeconom.2011.01.003 . hal-00815563

\section{HAL Id: hal-00815563 \\ https://hal.science/hal-00815563}

Submitted on 19 Apr 2013

HAL is a multi-disciplinary open access archive for the deposit and dissemination of scientific research documents, whether they are published or not. The documents may come from teaching and research institutions in France or abroad, or from public or private research centers.
L'archive ouverte pluridisciplinaire HAL, est destinée au dépôt et à la diffusion de documents scientifiques de niveau recherche, publiés ou non, émanant des établissements d'enseignement et de recherche français ou étrangers, des laboratoires publics ou privés. 


\section{Accepted Manuscript}

Estimation of fractional integration under temporal aggregation

Uwe Hassler

PII:

S0304-4076(11)00014-5

DOI:

10.1016/j.jeconom.2011.01.003

Reference: ECONOM 3443

To appear in: Journal of Econometrics

Received date: 17 March 2010

Revised date: 22 January 2011

Accepted date: 24 January 2011

Please cite this article as: Hassler, U., Estimation of fractional integration under temporal aggregation. Journal of Econometrics (2011), doi:10.1016/j.jeconom.2011.01.003

This is a PDF file of an unedited manuscript that has been accepted for publication. As a service to our customers we are providing this early version of the manuscript. The manuscript will undergo copyediting, typesetting, and review of the resulting proof before it is published in its final form. Please note that during the production process errors may be discovered which could affect the content, and all legal disclaimers that apply to the journal pertain. 


\title{
Estimation of Fractional Integration under Temporal Aggregation
}

\author{
Uwe Hassler* \\ Goethe University Frankfurt ${ }^{\dagger}$
}

January 25, 2011

\begin{abstract}
A result characterizing the effect of temporal aggregation in the frequency domain is known for arbitrary stationary processes and generalized for difference-stationary processes here. Temporal aggregation includes cumulation of flow variables as well as systematic (or skip) sampling of stock variables. Next, the aggregation result is applied to fractionally integrated processes. In particular, it is investigated whether typical frequency domain assumptions made for semiparametric estimation and inference are closed with respect to aggregation. With these findings it is spelled out, which estimators remain valid upon aggregation under which conditions on bandwidth selection.
\end{abstract}

Keywords: long memory, difference-stationarity, cumulating time series, skip sampling, closedness of assumptions

* An earlier version of this paper was written while visiting the University of California San Diego and was presented at Texas A\&M University, Universidad Carlos III de Madrid, Institute for Advanced Studies, Vienna, and the 3rd ETSERN Meeting, Nottingham. I am grateful to Patrik Guggenberger, Joon Park, Benedikt Pötscher, Philippe Soulier, Jim Stock, Yixiao Sun, and Carlos Velasco for support and many insights. Moreover, I thank two anonymous referees and Peter Robinson for very helpful comments.

${ }^{\dagger} \mathrm{RuW}$, Grueneburgplatz 1, 60323 Frankfurt, Germany, tel: +49-69-798-34762, fax: +49-69-798-35014. Email: hassler@wiwi.uni-frankfurt.de 
JEL classification: C14 (Semiparametric and Nonparametric Methods), C22 (Time-Series Models), C82 (Methodology for Collecting, Estimating, and Organizing Macroeconomic Data)

\section{Introduction}

Determining inflation persistence is a prominent issue when it comes to forecasting (Stock and Watson, 2007), or when monetary policy recommendations are at stake, see e.g. Mishkin (2007). The effect of temporal aggregation on inflation persistence has recently been studied by Paya, Duarte, and Holden (2007). Fractional integration is one model for inflation persistence that can be traced back to Hassler and Wolters (1995) or Baillie, Chung, and Tieslau (1996). The question how aggregation and persistence interact is of interest beyond inflation, and has troubled applied economists for a long time, see Christiano, Eichenbaum, and Marshall (1991) for empirical evidence in the context of the permanent income hypothesis and Rossana and Seater (1995) for a representative set of economic time series. Using fractionally integrated models, Chambers (1998) found with macroeconomic series that the empirical degree of integration may depend on the level of temporal aggregation, see also Diebold and Rudebusch (1989) or Tschernig (1995). In empirical finance, too, one of the core issues with respect to realized volatility is optimal sampling, see e.g. Ait-Sahalia, Mykland, and Zhang (2005) and the results by Drost and Nijman (1993).

In this paper we understand by temporal aggregation both: systematic sampling (or skip sampling) of stock variables where only every $p$ th data point is observed, and summation of flow variables where neighbouring observations are cumulated to determine the total flow. Econometricians have devoted their attention to both types of temporal aggregation for decades, see Silvestrini and Veredas (2008) for a recent survey. Early results for autoregressive moving-average (ARMA) models were obtained by Brewer (1973) and Weiss (1984). A treatment of integrated (of order one) ARIMA models was provided by Wei (1981) and Stram and Wei (1986), for skip sampling 
and cumulating, respectively. In particular, skip sampling can be embedded in the more general problem of missing observations, see Palm and Nijman (1984) for an investigation of dynamic regression models. Aspects of forecasting have been addressed by Lütkepohl (1987) and Lütkepohl (2009), while Marcellino (1999) deals with cointegration and causality under aggregation. Moreover, the potential interaction of seasonal integration and unit roots at frequency zero due to temporal aggregation was studied by Granger and Siklos (1995), see also Pons (2006). In fact, there is a literature on "span versus frequency" when it comes to testing the null hypothesis of a unit root, which started with Shiller and Perron (1985) and came to a preliminary end with Chambers (2004).

Notwithstanding the vast amount of papers on temporal aggregation, little attention has been paid to effects in the frequency domain, notable exceptions being Drost (1994) and Souza (2003). In the frequency domain, temporal aggregation is accompanied by the so-called aliasing effect, which is well known under discrete-time sampling from a continuous-time process, see e.g. Hansen and Sargent (1983). For the special case of fractional integration, spectral results have been obtained by Chambers (1998), Hwang (2000), Tsai and Chan (2005b), and Souza (2005). Further, Chambers (1996) and Tsai and Chan (2005a) cover the related case of discrete-time sampling from a continuous-time long memory process, while Souza $(2007,2008)$ focusses on the effect of temporal aggregation on widely used memory estimators.

We add two aspects to this literature: a general characterization of time aggregation in the frequency domain for processes that become stationary only after differencing $r$ times for some natural number $r$, and an investigation, which semiparametric estimators of fractionally integrated models retain their consistency and limiting normality under aggregation. In greater detail our contributions are the following. We draw from the literature results on aliasing and moving-averaging in case of temporal aggregation of arbitrary stationary processes (Lemma 1 and 2), and we combine these lemmae to characterize the frequency domain effect of temporal aggregation 
for processes that become stationary only after integer differencing $r$ times, $r=0,1,2, \ldots$ (Proposition 1 ). Next, the aggregation results are applied to fractionally integrated processes. In particular, we investigate whether typical assumptions on fractionally integrated processes, which are made in the literature to obtain consistency or limiting normality of semiparametric estimators, are closed with respect to aggregation. In other words: if $\left\{z_{t}\right\}$ satisfies a set of assumptions used to prove properties of some estimator or test, does the temporal aggregate fulfill them, too? Differing findings are obtained for cumulating of flow data (Proposition 2), skip sampling of stocks (Proposition 3), and for the case of generalized fractional integration where the singularity may occur at frequencies different from zero (Proposition 4). In a couple of remarks we discuss as consequences for applied work, which estimators remain valid upon aggregation (under which conditions on the bandwidth choice).

The rest of this paper is organized as follows. Section 2 treats the general aggregation effect in terms of spectral densities. In Section 3, the aggregation results are applied to the semiparametric estimation of the memory parameter of fractional integration. The last section contains a more detailed non-technical summary. Proofs are relegated to the Appendix.

\section{Aggregation in the frequency domain}

For sequences $\left\{a_{j}\right\}$ and $\left\{b_{j}\right\}$, let $a_{j} \sim b_{j}$ denote $a_{j} / b_{j} \rightarrow 1$ as $j \rightarrow \infty$, while for functions, $a(x) \sim b(x)$ is short for $a(x) / b(x) \rightarrow 1$ as $x \rightarrow 0$. Further, $a(x)=O\left(x^{c}\right)$ means that $a(x) x^{-c}$ is bounded as $x \rightarrow 0$, while $a(x)=o\left(x^{c}\right)$ signifies $a(x) x^{-c} \rightarrow 0$. First-order derivatives are given as $a^{\prime}(x)$. Finally, let $\mathbb{Z}$ stand for the set of all integers.

\subsection{Notation and assumptions}

Let $\left\{z_{t}\right\}, t=1,2, \ldots, T$, denote some time series to be aggregated over $p$ periods. The aggregate is constructed for the new time scale $\tau$. In case 
of stock variables, aggregation or systematic sampling means skip sampling where only every $p$ 'th data point is observed,

$$
\dot{z}_{\tau}:=z_{p \tau}, \quad \tau=1,2, \ldots,
$$

where for the rest of the paper $p \geq 2$ is a finite integer. Flow variables are aggregated by cumulating $p$ neighbouring observations that do not overlap to determine the total flow over $p$ sub-periods,

$$
\begin{aligned}
\widetilde{z}_{\tau} & :=z_{p \tau}+z_{p \tau-1}+\ldots+z_{p(\tau-1)+1} \\
& =S_{p}(L) z_{p \tau}, \quad \tau=1,2, \ldots,
\end{aligned}
$$

where $S_{p}(L):=1+L+\cdots+L^{p-1}$ is the moving average polynomial of degree $p$ in the usual lag operator $L$. Hence, $\left\{\widetilde{z}_{\tau}\right\}$ is obtained by skip sampling the overlapping moving average process $\left\{S_{p}(L) z_{t}\right\}$.

Clearly, many economic variables are not stationary. It is often assumed that the basic variable $\left\{z_{t}\right\}$ is given by integration over stationary increments,

$$
z_{t}=z_{0}+\sum_{i=1}^{t} y_{i}, \quad t=1,2, \ldots, T .
$$

If $\left\{y_{t}\right\}$ is a stationary fractionally integrated process of order $d, d<0.5$, as defined in a subsequent section, then the partial sum process $\left\{z_{t}\right\}$ is sometimes called fractionally integrated (of order $\delta=1+d$ ) of "type I", see Marinucci and Robinson (1999) and Robinson (2005). Some economic variables are even considered as integrated of order 2. Therefore, we allow for stationarity and different degrees of nonstationarity at the same time. It is maintained for some natural number $r \in\{0,1,2, \ldots\}$ that the process $\left\{z_{t}\right\}$ solves the following difference equation with $\Delta=1-L$ :

$$
\Delta^{r} z_{t}=y_{t}, \quad t=1,2, \ldots, T .
$$

Note that differencing changes the status of stock series: While log-prices $p_{t}=\log P_{t}$ are stocks, the inflation rate $\pi_{t}=\Delta p_{t}$ is a flow variable.

To fully specify the potentially nonstationary processes from (3), we have to add assumptions on $\left\{y_{t}\right\}$. Our results will hold for any stationary process 
$\left\{y_{t}\right\}$ with integrable spectral density $f_{y}$. Since $f_{y}$ is an even and $2 \pi$-periodic function, the definition of the spectral density can be extended to the whole real range, and we focus on the interval $[0, \pi]$ in the following assumption.

Assumption 1 The process $\left\{y_{t}\right\}, t \in \mathbb{Z}$, is covariance stationary with integrable spectral density $f_{y}(\lambda)$ on $\Pi$, where $\Pi=[0, \pi]$ if $f_{y}$ is well defined on the whole interval, or $\Pi=[0, \pi] \backslash\left\{\lambda^{*}\right\}$ if $f_{y}$ has a singularity at some frequency $\lambda^{*} \in[0, \pi]$.

Note that $f_{y}$ does not have to exist everywhere. A singularity at $\lambda^{*}$ might come from (generalized) fractional integration with long memory, see (12) below. In fact, we might allow for $k$ singularities (having e.g. so-called $k$-factor Gegenbauer processes in mind, see Woodward, Cheng, and Gray, 1998). Further, we stress that $f_{y}(0)=0$ is not excluded. This covers the particular case of over-differencing. Assume e.g. that no differencing is required to obtain stationarity, but $\left\{z_{t}\right\}$ is differenced in practice. This case is dealt with by $r=1$ in (3) with the assumption that $\left\{y_{t}\right\}$ is over-differenced.

To set the scene for the next subsection, we define the lag operator $\mathcal{L}$ operating on the aggregate time scale $\tau$, such that $\mathcal{L}=L^{p}$ with $L$ operating on $t$ (see e.g. Wei, 1990, Ch.16). Let $\nabla=1-\mathcal{L}$ stand for the differences of the new time scale $\tau$. In case that $r \geq 1$ in (3), we will study the effect of first aggreating and then differencing. The spectral densities of the differenced aggregates $\left\{\nabla^{r} \dot{z}_{\tau}\right\}$ and $\left\{\nabla^{r} \widetilde{z}_{\tau}\right\}$ are denoted as $\dot{f}_{\nabla^{r} z}(\lambda)$ and $\widetilde{f}_{\nabla^{r} z}(\lambda)$, respectively. For $r=0$, we have $z_{t}=y_{t}$ and $\dot{f}_{y}(\lambda)$ or $\tilde{f}_{y}(\lambda)$ represent the spectra of the stationary aggregates $\left\{\dot{y}_{\tau}\right\}$ and $\left\{\widetilde{y}_{\tau}\right\} .{ }^{1}$

\subsection{Result and discussion}

The main effect in the frequency domain is the so-called aliasing effect that arises from skip sampling. Since cumulation of non-overlapping data can be

\footnotetext{
${ }^{1}$ Sometimes stock variables are aggregated by averaging over $p$ non-overlapping observations, $\left\{\bar{z}_{\tau}\right\}$, such that $p$ sub-periods are replaced by the mean of $p$ values. Obviously this is directly connected to cumulation from $(2), \bar{z}_{\tau}:=\widetilde{z}_{\tau} / p$. Let the spectrum of the differenced aggregate $\left\{\nabla^{r} \bar{z}_{\tau}\right\}$ be denoted as $\bar{f}_{\nabla^{r} z}(\lambda)$. There is no need to address the case of averaging separately since it holds $\bar{f}_{\nabla^{r} z}(\lambda)=\widetilde{f}_{\nabla^{r} z}(\lambda) / p^{2}$.
} 
reduced to skip sampling a moving average, the effect will be present also with flow data. Therefore, we first pin down the aliasing effect. The following finding for stationary processes is essentially due to Drost (1994, Lemma 2.1). We highlight his result as a lemma, since many authors seem to be not aware of it, see e.g. Chambers (1998), Hwang (2000), Souza (2005), and Tsai and Chan (2005b), although an equivalent representation can be found in Souza (2003, Theo. 1).

Lemma 1 (Aliasing) Let $\left\{z_{t}\right\}$ from (3) with $r=0$ equal $\left\{y_{t}\right\}$ with Assumption 1, and assume that its spectral density $f_{y}$ is bounded at $(\lambda+2 \pi j) / p$, $j=1, \ldots,(p-1)$. It then holds for the spectral density of the skip sampled aggregate over $p$ periods, $\left\{\dot{y}_{\tau}\right\}$ :

$$
\dot{f}_{y}(\lambda)=\frac{1}{p} \sum_{j=0}^{p-1} f_{y}\left(\frac{\lambda+2 \pi j}{p}\right) .
$$

The summation over the frequencies $\frac{\lambda+2 \pi j}{p}, j=0,1, \ldots, p-1$, in Lemma 1 corresponds to the well known aliasing effect that occurs when observing a continuous-time process at discrete points in time, see e.g. Hansen and Sargent (1983), or the discussion in Priestley (1981, p.224, p.506): Cycles of frequency $\frac{\lambda+2 \pi j}{p}$ in the basic data become cycles of frequency $\lambda+2 \pi j$ upon skip sampling, and are hence indistinguishable from $\lambda$.

A second effect that will be present in case of cumulation on top of aliasing is the transfer function of the moving average filter $S_{p}(L)$, see (2). This effect also shows up when considering differenced aggregates with $\nabla=\left(1-L^{p}\right)=$ $S_{p}(L)(1-L)$, and it is characterized in the following lemma. The required transfer function is given e.g. in Priestley (1981, p.270), where $T_{j}(\lambda)$ is proportional to the so-called Fejér kernel, see e.g. Priestley (1981, p.401, p.418) for a discussion. 
Lemma 2 (Transfer function of $S_{p}(L)$ ) The transfer function $\left|S_{p}\left(e^{i \cdot}\right)\right|^{2}$ evaluated at $(\lambda+2 \pi j) / p$ for $j=0, \ldots, p-1$ is equal to

$$
T_{j}(\lambda):=\frac{\sin ^{2}\left(\frac{\lambda}{2}\right)}{\sin ^{2}\left(\frac{\lambda+2 \pi j}{2 p}\right)}, \quad \lambda>0,
$$

where $T_{j}(\lambda)$ is continuously differentiable with

$$
\begin{gathered}
T_{0}(\lambda)=p^{2}+O\left(\lambda^{2}\right), \quad T_{j}(\lambda)=O\left(\lambda^{2}\right), \quad j=1, \ldots, p-1, \\
T_{j}^{\prime}(\lambda)=O(\lambda), \quad j=0, \ldots, p-1,
\end{gathered}
$$

as $\lambda \rightarrow 0$.

Now, it is straightforward to prove the general result.

Proposition 1 Let $\left\{y_{t}\right\}$ be from Lemma 1, and let $\left\{\Delta^{r} z_{t}\right\}$ equal $\left\{y_{t}\right\}, r=$ $0,1,2, \ldots$ It then holds for the spectral densities of the differences of the aggregates of $\left\{z_{t}\right\}$

a) in case of skip sampling $\left(\nabla^{r} \dot{z}_{\tau}\right)$ :

$$
\dot{f}_{\nabla^{r} z}(\lambda)=\frac{1}{p} \sum_{j=0}^{p-1} f_{y}\left(\frac{\lambda+2 \pi j}{p}\right)\left[T_{j}(\lambda)\right]^{r},
$$

b) and in case of cumulating $\left(\nabla^{r} \widetilde{z}_{\tau}\right)$ :

$$
\widetilde{f}_{\nabla^{r} z}(\lambda)=\frac{1}{p} \sum_{j=0}^{p-1} f_{y}\left(\frac{\lambda+2 \pi j}{p}\right)\left[T_{j}(\lambda)\right]^{r+1},
$$

where $T_{j}(\lambda), j=0,1, \ldots,(p-1)$, are from Lemma 2.

Proof See Appendix.

It seems advisable to discuss the proposition with a couple of comments. 
First, the cumulated stationary aggregate, $\widetilde{f}_{\nabla^{0} z}(\lambda)=\widetilde{f}_{y}(\lambda)$, is subject to aliasing, too, simply because $\left\{\widetilde{y}_{\tau}\right\}$ is constructed from skip sampling a moving average. In this case, however, aliasing is superimposed by the factors $T_{j}(\lambda)$ due to the moving average filter $S_{p}(L)$. Consequently, at frequency zero the aliased frequencies are squelched out, and it holds in case of cumulation $(\lambda \rightarrow 0)$

$$
\widetilde{f}_{y}(\lambda) \sim p f_{y}\left(\frac{\lambda}{p}\right), \quad \tilde{f}_{y}^{\prime}(\lambda) \sim f_{y}^{\prime}\left(\frac{\lambda}{p}\right)
$$

In particular, the slope of $f_{y}(\lambda)$ around frequency zero is inherited by $\widetilde{f}_{y}$. A similar effect shows up for spectra from differences, $r \geq 1$.

Second, an immediate consequence of Proposition 1 is that differencing and temporal aggregation are not exchangeable without required modification. Below eq. (3), we noted that differencing stock variables yields flow data. Consequently, for $r=1$, when comparing the spectral densities of the differenced aggregates $(\nabla z)$ with the aggregates of the stationary differences $(\Delta z)$, we find that differencing skip sampled stock data has the same effect as cumulating the differences (flows):

$$
\dot{f}_{\nabla z}(\lambda)=\widetilde{f}_{\Delta z}(\lambda) \neq \dot{f}_{\Delta z}(\lambda) \text {, and } \widetilde{f}_{\nabla z}(\lambda) \neq \widetilde{f}_{\Delta z}(\lambda) .
$$

Third, Proposition 1 contains a unifying framework for several familiar results. The result a) for $r=0$ of course reproduces the original Lemma 1 . The result b) for $r=0$ is from Drost (1994, Lemma 2.2), while an equivalent representation can be found again in Souza (2003). For the special case of fractionally integrated ARMA processes Tsai and Chan (2005b, Theo. 1(a)) provide equivalent results under cumulation (Proposition $1 \mathrm{~b}$ )). Notice that they have to spend more than two pages of technically involved derivations to establish their special case, while our more general result follows in a very straightforward manner from Lemmae 1 and 2.

Proposition 1 will enable us to investigate systematically which properties of the basic process are inherited by the aggregates. Such properties are called closed in the following sense. 
Definition 1 A set of assumptions on some process $\left\{z_{t}\right\}$ is called closed with respect to temporal aggregation (skip sampling or cumulating), if $\left\{\dot{z}_{\tau}\right\}$ or $\left\{\widetilde{z}_{\tau}\right\}$, respectively, satisfy the same set of assumptions for any finite positive integer $p \geq 2$, too.

For practical purposes procedures with properties established under assumptions that are closed with respect to aggregation are desirable, because in most practical situations a "true" frequency of the DGP is not known or does not exist. Most economic and financial time series have to be considered as aggregates. And a statistical procedure relying on a set of assumptions $\mathcal{A}$ cannot be safely applied to an aggregate, unless $\mathcal{A}$ is closed with respect to temporal aggregation. With Proposition 1 at hand we will now discuss closedness and lack thereof of certain general assumptions about fractionally integrated processes.

\section{Fractional integration}

\subsection{Assumptions}

Let us consider the fractionally integrated process $\left\{y_{t}\right\}$ constructed from the filter $(1-L)^{-d}$ with the usual expansion,

$$
y_{t}=(1-L)^{-d} e_{t}, \quad \text { with }|d|<0.5,
$$

where the short memory component $\left\{e_{t}\right\}$ is a stationary process with spectral density $f_{e}$. For $\left\{y_{t}\right\}$ it holds $f_{y}(\lambda)=\left|1-e^{i \lambda}\right|^{-2 d} f_{e}(\lambda)$. Equivalently (because $\left|1-e^{i \lambda}\right|^{-2 d}=\lambda^{-2 d}(1+o(1))$ fractional integration is characterized through the assumption

$$
f_{y}(\lambda)=\lambda^{-2 d} f_{e}(\lambda), \quad|d|<0.5
$$

Papers on semiparametric inference of long memory typically assume that the observed process has a spectral density like in (6) where the short memory component $f_{e}$ is characterized by assumptions $\mathcal{A}$ as weak as possible. We consider typical spectral assumptions next. 
Assumption 2 Let $\mathcal{A}$ be a set of assumptions for $f_{y}(\lambda)=\lambda^{-2 d} f_{e}(\lambda),|d|<$ 0.5 , including

(A0) $f_{e}$ is bounded and bounded away from zero at frequency $\lambda=0$;

(A1) for some $\beta \in(0,2]$ it holds

$$
f_{e}(\lambda)=f_{e}(0)+O\left(\lambda^{\beta}\right), \quad \lambda \rightarrow 0
$$

(A2) $f_{e}$ has a finite first derivative $f_{e}^{\prime}$ in a neighbourhood $(0, \epsilon)$ of zero, and

$$
f_{e}^{\prime}(\lambda)=O\left(\lambda^{-1}\right), \quad \lambda \rightarrow 0
$$

(A3) $f_{e}$ has a finite first derivative $f_{e}^{\prime}$ at $\lambda=0$.

The first assumption (A0) that $f_{e}(0)$ is bounded and positive is minimal and common to all papers in order to identify $d$ from (6). Next, assumption (A1) imposes a rate of convergence on (6) characterizing the smoothness of the short memory component $f_{e}$ around zero. If $\left\{e_{t}\right\}$ is ARMA, then $\beta=2$. With $m$ denoting the bandwidth of semiparametric estimators and $T$ standing for the sample size, the parameter $\beta$ controls the rate the bandwidth has grow with through the following condition:

$$
\frac{1}{m}+\frac{m^{1+2 \beta}(\log m)^{2}}{T^{2 \beta}} \rightarrow 0
$$

implying $m=o\left(T^{2 \beta /(1+2 \beta)}\right)$. Assumption (A1) is widely used to establish not only consistency, but also limiting normality of semiparametric memory estimators, see e.g. Robinson (1995a, Ass. 1'), Robinson (1995b, Ass. 1), Velasco (1999a, Ass. 2), Velasco (1999b, Ass. 1), Shimotsu and Phillips (2005, Ass. 1'), and Shimotsu (2010, Ass. $\left.1^{\prime}\right) .^{2}$ While this assumption implies that $f_{e}$ is continuous on $(0, \epsilon)$, some results require that the derivative $f_{e}^{\prime}$ exists in a neighbourhood of the origin, even if it may diverge at appropriate rate as getting close to zero, see Assumption (A2). Although put

\footnotetext{
${ }^{2}$ Allowing for tapered data, a slightly stronger, parametric version of assumption (A1) is required, $f_{e}(\lambda)=b_{0}+b_{1} \lambda^{\beta}+o\left(\lambda^{\beta}\right)$, see e.g. Velasco (1999a, Ass. 8), Velasco (1999b, Ass. 2), Hurvich and Chen (2000, Ass. 1), and also Abadir, Distaso, and Giraitis (2007, eq. $(2.23))$.
} 
slightly differently such an assumption is found again in Robinson (1995a, Ass. 2), Velasco (1999a, Ass. 3), and Shimotsu and Phillips (2005, Ass. 2) or Shimotsu (2010, Ass. 2) when establishing consistency of the local Whittle (LW) estimator and the so-called exact LW estimator, respectively. ${ }^{3} \mathrm{~A}$ related but slightly weaker condition is employed in Robinson (1994, Ass. 4) and Lobato and Robinson (1996, (C2)) to determine optimal spectral bandwidth rates and limiting properties of the averaged periodogram estimator, respectively. Other papers assume a stronger degree of smoothness of $f_{e}$ at frequency zero in that they demand the first derivative $f_{e}^{\prime}(0)$ to be finite (or even zero), which is our assumption (A3). Hurvich, Deo, and Brodsky (1998) for instance assume $f_{e}^{\prime}(0)=0$ when deriving the asymptotic mean squared error and limiting distribution of the log-periodogram regression (LPR) by Geweke and Porter-Hudak (1983), while Andrews and Guggenberger (2003) discuss properties of a bias-reduced version under a smoothness assumption requiring $f_{e}^{\prime}(0)$ to exist, see also Guggenberger and Sun (2006). Under similar assumptions Andrews and Sun (2004) improved on the LW estimator.

Since the following results are obtained under temporal aggregation we need spectral assumptions for $\lambda>0$ due to the aliasing effect. We require that the spectral density is "well behaved" at multiples of the so-called Nyquist frequency $2 \pi / p$, see Proposition 1 . The usual long memory literature not addressing the aggregation issue does not need Assumption 3. Souza (2007, Cond. 3 and 9), however, when addressing memory estimation under cumulation formulates very similar assumptions.

Assumption 3 The process $\left\{y_{t}\right\}$ from Assumption 1 has a spectral density $f_{y}(\lambda)$, which at frequencies $2 \pi j / p, j=1, \ldots,(p-1)$, is bounded, bounded away from zero and continuously differentiable with derivative $f_{y}^{\prime}$.

\subsection{Cumulation of flow variables}

It has been documented empirically that cumulation of flow variables will affect memory estimation in finite samples, see e.g. Diebold and Rudebusch

\footnotetext{
${ }^{3}$ See also the assumption $\left|f_{e}^{\prime}(\lambda)\right| \leq c \lambda^{-1}$ for $\lambda>0$ in Moulines and Soulier (1999, Ass. 2 ), and similar although slightly weaker in Soulier (2001, Ass. 1).
} 
(1989), Tschernig (1995), and Chambers (1998). Experimentally, a finite sample bias due to cumulation has been reported by Teles, Wei, and Crato (1999) and Souza (2007). In this subsection, we address the asymptotic properties of some well-known semiparametric memory estimators for finite $p$; the effect of increasing aggregation level $(p \rightarrow \infty)$ on cumulation has been investigated by Man and Tiao (2006) in the time domain and by Tsai and Chan (2005b) with spectral methods.

Let us briefly discuss the cumulation of stationary flow variables, $z_{t}=y_{t}$. From (4) it is obvious that a zero or just as well a singularity of $f_{y}$ at frequency zero is inherited by $\tilde{f}_{y}$, and the spectral slope of $\left\{y_{t}\right\}$ at frequency zero is carried over to the aggregate $\left\{\widetilde{y}_{\tau}\right\}$, or in other words: assumptions about the spectral slope of stationary processes at frequency zero are closed with respect to cumulating. This confirms the finding by Chambers (1998), Hwang (2000), and Souza (2005) that the order of fractional integration at the origin is maintained under cumulated aggregation of flow variables. More formally, it holds the following result for the stationary and nonstationary case at the same time; the result for $r=0$ was obtained as part of the proof in Souza (2007, p.721).

Proposition 2 Let $\left\{\Delta^{r} z_{t}\right\}$ with $r=0,1, \ldots$ equal $\left\{y_{t}\right\}$ with spectral density as in (6) satisfying Assumptions 1 and 3. It then holds for the spectral density of the differences $\nabla^{r}$ of $\left\{\widetilde{z}_{\tau}\right\}$

$$
\tilde{f}_{\nabla^{r} z}(\lambda)=\lambda^{-2 d} \widetilde{\varphi}_{r}(\lambda)
$$

with

$$
\widetilde{\varphi}_{r}(\lambda)=f_{e}\left(\frac{\lambda}{p}\right)\left(p^{2 d+2 r+1}+O\left(\lambda^{2}\right)\right)+\lambda^{2 d} \widetilde{R}_{r}(\lambda),
$$

where $\widetilde{R}_{r}(\lambda)$ is differentiable in a neighbourhood of $\lambda=0$ with

$$
\widetilde{R}_{r}(\lambda)=O\left(\lambda^{2 r+2}\right) \quad \text { and } \quad \widetilde{R}_{r}^{\prime}(\lambda)=O\left(\lambda^{2 r+1}\right), \quad \lambda \rightarrow 0 .
$$

Proof See Appendix. 
We want to spell out explicitly the closedness of the conditions from Assumption 2. From Proposition 2 it follows for $|d|<0.5$ :

under (A0): $\widetilde{\varphi}_{r}(0)=p^{2 d+2 r+1} f_{e}(0)$;

under (A1): $\widetilde{\varphi}_{r}(\lambda)=p^{2 d+2 r+1}\left(f_{e}(0)+O\left(\lambda^{\min (\beta, 2 d+2 r+2)}\right)\right)$;

under (A2): $\widetilde{\varphi}_{r}^{\prime}(\lambda)=O\left(\lambda^{-1}\right)$;

under (A3): $\widetilde{\varphi}_{r}^{\prime}(0)=p^{2 d+2 r} f_{e}^{\prime}(0)$.

For $r \geq 1$, the smoothness parameter $\beta$ from (A1) of $f_{e}$ carries over to $\widetilde{\varphi}_{r}$, and this holds true for $r=0$ with $d \geq 0$, too. For $r=0$ with $d<$ 0 , Assumption (A1) is still closed in that there exists a new smoothness parameter $\min (\beta, 2 d+2) \in(0,2]$. Note that the parametric version of (A1) given in footnote 2 is closed as well. We want to discuss consequences with respect to statistical inference in two remarks.

REMARK A For $r=0$, Souza (2007) proved that the LW and the LPR estimators retain the limiting normal distribution under cumulation of stationary series. To that end he showed Proposition 1 for $r=0$ and established the closedness of some further sufficient conditions $\left(\left\{e_{t}\right\}\right.$ is a linear sequence with certain moment and regularity conditions). In addition, we want to highlight Assumption (A1) for the stationary case:

$$
\widetilde{\varphi}_{0}(\lambda)=p^{2 d+1} f_{e}(0)+O\left(\lambda^{\min (\beta, 2 d+2)}\right), \quad \lambda \rightarrow 0 .
$$

Hence, for $d<0$ it may happen that $\min (\beta, 2 d+2)<\beta$, implying a slower rate for the bandwidth according to (7) after cumulation: $m=$ $o\left(T^{(4 d+4) /(5+4 d)}\right)$.

Remark B Velasco (1999a, Theo. 3) and Velasco (1999b, Theo. 3) prove the limiting normal distribution of the LW and the LPR estimators, respectively, when applied to nonstationary levels integrated of order $0.5<\delta<0.75$. More generally, Abadir et al. (2007, Coro. 2.1) showed that the so-called fully extended LW has a limiting normal distribution when applied to nonstationary levels integrated of any order $\delta>0.5$. In all three papers the main assumption is (A1), which turns out to be closed with respect to cumulation of difference-stationary series $(r \geq 1)$. Further assumptions they 
require (again, $\left\{e_{t}\right\}$ is a linear sequence with certain moment and regularity conditions) have been established in Souza (2007), see Remark A. Hence, the asymptotic results by Velasco (1999a,b) or Abadir et al. (2007) for nonstationary series remain valid after cumulation.

\section{3 $\quad$ Skip sampling}

Souza and Smith (2002) provide bias approximations for some semiparametric estimators that are well supported experimentally. Considerable finite sample biases are found due to skip sampling. Here, we add asymptotic insights by discussing closedness and lack thereof of Assumption 2 under skip sampling. We start with nonstationary processes because we know from Proposition 1 that $\dot{f}_{\nabla^{r} z}=\widetilde{f}_{\nabla^{r-1} z}$. Consequently, the results for skip sampling under $r \geq 1$ are contained in Proposition 2 already! Therefore, Remark B carries over to skip sampling as follows.

REMARK C The limiting normality established in Velasco (1999a, Theo. 3), Velasco (1999b, Theo. 3), and Abadir et al. (2007, Coro. 2.1) continues to hold when applied to skip sampled nonstationary levels integrated of order $\delta$ as in Remark B.

Now, we turn to the stationary case, $r=0$. Before showing a further proposition, we recollect some findings with respect to Assumption (A0) from the literature.

Let us consider a stationary process $\left\{y_{t}\right\}$ with $f_{y}(0)=0$. Proposition 1 a) yields $\dot{f}_{y}(0)=p^{-1} \sum_{j=0}^{p-1} f_{y}(2 \pi j / p)$. Hence, the assumption $f_{y}(0)=$ 0 is not closed with respect to skip sampling except for the unlikely case where $f_{y}(2 \pi j / p)=0$ for $j=1, \ldots, p-1$. This has first been observed by Drost (1994, p. 16), and it corrects differing claims made in Chambers (1998) and Hwang (2000), see also the elucidating discussion by Souza (2005): Integration of order $d$ in the sense of $(6)$ is not closed under skip sampling for $d<0$. This is a puzzling result at first glance, since fractional processes are known to be self-similar in that stretching the time scale leaves distributional 
properties unchanged upon rescaling the process, see e.g. Mandelbrot and van Ness (1968). In fact, for ARFIMA processes it holds for $|d|<0.5$ that

$$
E\left(\dot{y}_{\tau} \dot{y}_{\tau+h}\right)=E\left(y_{t} y_{t+p h}\right) \sim C(p h)^{2 d-1}, \quad h \rightarrow \infty
$$

for some constant $C$. Hence, the hyperbolic decay of the autocovariance is inherited by the skip sampled process irrespective of the sign of $d$, while the power law in (6) is lost for $d<0$. However, this lack of closedness is of little practical concern. Note that negative orders of integration typically arise only after differencing, and differencing a stock variable results in a flow series, which should be aggregated by cumulating, not by skip sampling.

Next, we provide a formal discussion of the effect of skip sampling stationary stock variables.

Proposition 3 Let $\left\{y_{t}\right\}$ be $I(d)$ with spectral density as in (6) satisfying Assumptions 1 and 3. It then holds for the spectral density of the skip sampled process

$$
\dot{f}_{y}(\lambda)=\lambda^{-2 d} \dot{\varphi}_{y}(\lambda) \quad \text { with } \quad \dot{\varphi}_{y}(\lambda)=p^{2 d-1} f_{e}\left(\frac{\lambda}{p}\right)+\lambda^{2 d} \dot{R}_{y}(\lambda),
$$

where $\dot{R}_{y}(\lambda)=\varphi_{1}+O(\lambda), 0<\varphi_{1}<\infty$, and $\dot{R}_{y}^{\prime}(\lambda)=O(1)$ as $\lambda \rightarrow 0$.

Proof See Appendix.

REMARK D The above discussion illustrates that the case $d<0$ may be ignored when talking about skip sampling. We now assume $d \geq 0$. From Proposition 3 it follows under (A1) with $\varphi_{0}=p^{2 d-1} f_{e}(0)$ :

$$
\dot{\varphi}_{y}(\lambda)=\varphi_{0}+O\left(\lambda^{\min (\beta, 2 d)}\right), \quad d>0 .
$$

Hence, Assumption (A1) is closed with $\alpha=\min (\beta, 2 d)$ only as long as $d \geq 0 .{ }^{4}$ Similarly, Assumption (A2) implies $\dot{\varphi}_{y}^{\prime}(\lambda)=O\left(\lambda^{-1}\right)$ as long as

\footnotetext{
${ }^{4}$ Strictly speaking, the case $d=0$ requires separate consideration with $\dot{\varphi}_{y}(\lambda)=p^{-1} f_{e}\left(\frac{\lambda}{p}\right)+\dot{R}_{y}(\lambda)=\varphi_{0}+\varphi_{1}+O\left(\lambda^{\beta}\right)+O(\lambda)=\varphi_{0}+\varphi_{1}+O\left(\lambda^{\min (\beta, 1)}\right)$.
} 
$d \geq 0$. Therefore, conditions by Robinson (1995a) used to prove consistency and limiting normality of the local Whittle estimator continue to hold after systematic sampling for $d \geq 0$. However, the order of integration $d$ may affect the required rate of divergence of the bandwidth $m$, see (7):

$$
m=o\left(T^{2 \alpha /(1+2 \alpha)}\right), \quad \alpha=\min (\beta, 2 d) .
$$

For values of $d$ close to zero with $\alpha=2 d$, this implies a very slow divergence of $m$, and hence a very slow convergence of some semiparametric estimator $\widehat{d}$ to the limiting distribution since the variance of $\widehat{d}$ is proportional to $1 / \mathrm{m}$.

REMARK E Note that Assumption (A3) is never closed with respect to skip sampling. The aggregated spectral density in (8) displays an unbounded derivative at the origin for all $d<0.5$ :

$$
\dot{\varphi}_{y}^{\prime}(\lambda)=p^{2 d-2} f_{e}^{\prime}\left(\frac{\lambda}{p}\right)+O\left(\lambda^{2 d-1}\right) .
$$

This means that sufficient conditions for consistency or limiting normality of the log-periodogram regression made by Hurvich et al. (1998) or Andrews and Guggenberger (2003) do not hold upon systematic sampling, which sheds some doubt on the use of the LPR in applied work. Notice, however, there is a trimmed version of the LPR by Robinson (1995b), where trimming means that the first $\ell$ harmonic frequencies are omitted from the regression. Robinson (1995b) assumes Assumptions (A1) and (A2), which are closed under skip sampling for $d \geq 0$. To ensure limiting normality of the trimmed LPR, Robinson (1995b, Ass. 6) requires with $\alpha$ from Remark D

$$
\frac{m^{1 / 2} \log m}{\ell}+\frac{\ell(\log T)^{2}}{m}+\frac{m^{1+1 / 2 \alpha}}{T} \rightarrow 0
$$

which obviously implies (9). While $m$ has again to diverge very slowly for small values of $d$, the trimming parameter $\ell$ has to diverge faster than $\sqrt{m}$, which makes appropriate choices of $\ell$ and $m$ a delicate matter in practice.

To shed further light on the effect of skip sampling it is elucidating to 
relate to a different strand of the literature. Let $\left\{x_{t}\right\}$ be a fractionally integrated process $\left\{y_{t}\right\}$ perturbed by some $I(0)$ process $\left\{u_{t}\right\}$,

$$
x_{t}=y_{t}+u_{t}
$$

where we assume that $\left\{u_{t}\right\}$ is independent of the unobservable process $\left\{y_{t}\right\}$. Given $\left\{y_{t}\right\}$ is fractionally integrated with (6) it holds in the frequency domain

$$
f_{x}(\lambda)=\lambda^{-2 d} f_{e}(\lambda)+f_{u}(\lambda)=\lambda^{-2 d} \varphi(\lambda)
$$

where the short memory component of the observable $\left\{x_{t}\right\}$ becomes

$$
\begin{aligned}
\varphi(\lambda) & =f_{e}(\lambda)+f_{u}(\lambda) \lambda^{2 d} \\
& \sim c_{0}+c_{1} \lambda^{2 d}, \quad \lambda \rightarrow 0,
\end{aligned}
$$

with $c_{0}=f_{e}(0)$ and $c_{1}=f_{u}(0)$. For $0<d$, the perturbed process $\left\{x_{t}\right\}$ is fractionally integrated of order $d$ where the short memory component $\varphi(\lambda)$ behaves like in case of skip sampling, cf. (8): skip sampling has in the frequency domain the same effect on long memory as adding noise. Therefore, methods tailored to the estimation of $d$ from $\left\{x_{t}\right\}$ in (10) are candidates for the estimation of $d$ from skip sampled long memory series. For that reason, a short and informal review of related work is provided to close down this subsection.

Most papers dealing with perturbed fractional integration (also called "long memory plus noise") are related to the so-called long memory stochastic volatility model (LMSV) introduced by Breidt, Crato, and de Lima (1998) or the FIEGARCH model by Bollerslev and Mikkelsen (1996). Such volatility models assume for return processes $\left\{r_{t}\right\}$ that

$$
\log r_{t}^{2}=\mu+y_{t}+\varepsilon_{t}
$$

where the perturbation term $\left\{\varepsilon_{t}\right\}$ is white noise. Sun and Phillips (2003) considered the more general model (10) under Gaussianity. They proposed an improved nonlinear version of the LPR estimator that accounts explicitly for the effect of perturbation. The bandwidth $m$ has to obey $m=o\left(T^{8 d /(8 d+1)}\right)$, 
which is less stringent than our condition (9) only if $\min (\beta, 2 d)<4 d$. Hurvich and Ray (2003) proposed a modification of the LW estimator adjusting explicitly for the noise effect of model (11); further refinements are provided by Hurvich, Moulines, and Soulier (2005) in that correlation between $y_{t}$ and $\varepsilon_{t}$ is allowed for. Finally, it should be noted that the so-called broadband log-periodogram regression by Moulines and Soulier (1999) remains valid for a Gaussian LMSV model, see Iouditsky, Moulines, and Soulier (1999).

\subsection{General fractional integration}

We now briefly touch the case where a singularity may occur at a frequency $\lambda^{*}$ different from zero:

$$
f_{y}(\lambda)=\left|\lambda^{*}-\lambda\right|^{-2 d} f_{e}(\lambda), \quad|d|<0.5, \quad \lambda^{*} \in[0, \pi] .
$$

A parametric model for such a spectral behaviour has been proposed by Gray, Zhang, and Woodward (1989), while the more recent literature focusses on a semiparametric approach only assuming that $f_{e}(\lambda)$ is bounded, bounded away from zero and twice continuously differentiable on $(0, \pi)$, see for instance Giraitis, Hidalgo, and Robinson (2001, Ass. A.1, A.1'), Hidalgo (2005, Cond. C.1), and Dalla and Hidalgo (2005, C1).

The following proposition focusses on the stationary case for convenience; the extension to $r \geq 1$ is obvious. It thus extends Proposition 2 and 3 in case that $r=0$.

Proposition 4 Let $\left\{y_{t}\right\}$ be $I(d)$ with spectral density as in (12) satisfying Assumptions 1 and 3. It then holds for the spectral density

a) of the skip sampled process

$$
\begin{gathered}
\dot{f}_{y}(\lambda)=\left|p \lambda^{*}-\lambda\right|^{-2 d} \dot{\varphi}_{y}^{*}(\lambda), \\
\dot{\varphi}_{y}^{*}(\lambda)=p^{2 d-1} f_{e}\left(\frac{\lambda}{p}\right)+\left|p \lambda^{*}-\lambda\right|^{2 d} \dot{R}_{y}(\lambda),
\end{gathered}
$$

where $\dot{R}_{y}$ is from Proposition 3; 
b) of the cumulated process

$$
\begin{gathered}
\widetilde{f}_{y}(\lambda)=\left|p \lambda^{*}-\lambda\right|^{-2 d} \widetilde{\varphi}_{y}^{*}(\lambda) \\
\widetilde{\varphi}_{y}^{*}(\lambda)=p^{2 d-1} f_{e}\left(\frac{\lambda}{p}\right) T_{0}(\lambda)+\left|p \lambda^{*}-\lambda\right|^{2 d} \widetilde{R}_{0}(\lambda),
\end{gathered}
$$

where $\widetilde{R}_{0}$ is from Proposition 2 , and $T_{0}(\lambda)$ from Lemma 2.

Proof Follows the lines of the proofs of Propositions 2 and 3 and is therefore omitted.

The final remark collects three comments.

REMARK F First, from Proposition 4 we observe that the singularity is shifted from frequency $\lambda^{*}$ to $p \lambda^{*}$ due to aggregation. If $p \lambda^{*}$ exceeds $\pi$, one may of course rescale due to periodicity and symmetry, and replace $p \lambda^{*}$ by $\lambda_{0} \in[0, \pi]:$

$$
\lambda_{0}=\left\{\begin{array}{ll}
2 k \pi-p \lambda^{*}, & \text { if } p \lambda^{*} \in((2 k-1) \pi, 2 k \pi] \\
p \lambda^{*}-2 k \pi, & \text { if } p \lambda^{*} \in(2 k \pi,(2 k+1) \pi]
\end{array} \quad, \quad k=1,2, \ldots\right.
$$

Second, contrary to (4), the moving average filter no longer annihilates the aliasing effect in case of cumulation (as $\lambda \rightarrow p \lambda^{*}$ ):

$$
\widetilde{f}_{y}(\lambda) \sim\left|p \lambda^{*}-\lambda\right|^{-2 d} p^{2 d-1} f_{e}\left(\lambda^{*}\right) T_{0}\left(p \lambda^{*}\right)+\widetilde{R}_{0}\left(p \lambda^{*}\right),
$$

where $\widetilde{R}_{0}\left(p \lambda^{*}\right)$ is positive in general. Hence, if $d<0$, a zero at $\lambda^{*}$ of $f_{y}$ is not reproduced by $\widetilde{f}_{y}$ at $p \lambda^{*}$, such that generalized fractional integration with $d<0$ is no longer closed under cumulation. This is not true, however, in the special case where $p \lambda^{*}$ is a multiple of $2 \pi$, because $\widetilde{R}_{0}$ is periodic, as one can see from its definition in the proof of Proposition 2. Third and most interestingly, in case $d \geq 0$, one may investigate whether assumptions about $f_{e}$ are inherited by the short memory components $\widetilde{\varphi}_{y}^{*}(\lambda)$ and $\dot{\varphi}_{y}^{*}(\lambda)$ of the aggregates. Both short memory components are not differentiable at frequency $p \lambda^{*}$, thus violating the typical assumption mentioned above made by Giraitis et al. (2001), Hidalgo (2005), and Dalla and Hidalgo (2005), and indeed by most other papers working under model (12). 


\section{Concluding remarks}

We characterize effects of cumulating flow variables as well as systematic sampling stock data of arbitrary stationary or difference-stationary processes in the frequency domain (Proposition 1). The results are applied to fractionally integrated processes of order $d$. In particular, we investigate whether typical assumptions on fractionally integrated processes, which are made in the literature to justify statistical semiparametric inference about $d$, are closed with respect to aggregation. That is we study whether assumptions that hold for basic data continue to hold for temporal aggregates, such that semiparametric methods like the log-periodogram regression (LPR) or the local Whittle (LW) estimator are justified for aggregates, too. It turns out (Proposition 2) that typical spectral assumptions made for semiparametric estimation are closed with respect to cumulating flow variables (or averaging non-overlapping stocks, see footnote 1). Hence, the semiparametric procedures discussed in Remark A for stationary data and in Remark B for the nonstationary case may be safely applied to those aggregates.

In case of skip sampling fractionally integrated stock variables matters are more complicated. The methods applied to nonstationary aggregates continue to hold (Remark C). In the stationary case, we conclude from Proposition 3 that certain properties that hold for the basic data can not be maintained for the aggregate, while other assumptions are closed as long as $d \geq 0$. More precisely, it turns out (Remark D) that the LW estimator can be applied to skip sampled aggregates, although the rate of divergence of the bandwidth may be influenced by $d$, such that the bandwidth selection may become a delicate problem in practice. A similar comment holds for the trimmed LPR (Remark E), while sufficient conditions for the conventional LPR do actually not carry over from the basic series to the aggregate. Note, that in practice such difficulties can be circumvented by aggregating stocks through averaging non-overlapping observations (see again footnote 1). Further, we reveal that skip sampling has the same spectral effect as adding noise to the data. Hence we suggest that shortcomings when estimating 
long memory under skip sampling may be alleviated using approaches tailored to cope with perturbed fractional integration or so-called long memory stochastic volatility models. An investigation how fruitful the many different procedures are in the presence of skip sampled long memory is beyond the present paper and has to be left for future research.

For the model of general fractional integration, where a spectral singularity may occur at a frequency different from zero, we treat cumulating and skip sampling in one go (Proposition 4). In Remark F it is concluded that an assumption that is ever-present in related literature is not closed with respect to aggregation. Consequently, when applying a model of general fractional integration one should always stick to the basic data and not work with aggregated time series.

\section{Appendix}

\section{Proof of Proposition 1}

We start to prove a). The case $r=0$ is covered by Lemma 1 . For $r \geq 1$ we observe:

$$
\nabla^{r} \dot{z}_{\tau}=\left[(1-L) S_{p}(L)\right]^{r} z_{p \tau}=\left[S_{p}(L)\right]^{r} y_{p \tau},
$$

i.e. $\left\{\nabla^{r} \dot{z}_{\tau}\right\}$ is obtained by skip sampling $\left\{\left[S_{p}(L)\right]^{r} y_{t}\right\}$. Consequently, by Lemma 1,

$$
\dot{f}_{\nabla^{r} z}(\lambda)=\frac{1}{p} \sum_{j=0}^{p-1} f_{y}\left(\frac{\lambda+2 \pi j}{p}\right)\left|S_{p}\left(\exp \left\{i \frac{\lambda+2 \pi j}{p}\right\}\right)\right|^{2 r},
$$

which is the required result with $T_{j}(\lambda)$ from Lemma 2. For cumulated series with $r \geq 0$ it holds in b):

$$
\nabla^{r} \widetilde{z}_{\tau}=\left[(1-L) S_{p}(L)\right]^{r} S_{p}(L) z_{p \tau}=\left[S_{p}(L)\right]^{r+1} y_{p \tau},
$$

i.e. $\left\{\nabla^{r} \widetilde{z}_{\tau}\right\}$ is obtained by skip sampling $\left\{\left[S_{p}(L)\right]^{r+1} y_{t}\right\}$. The result follows as in a), which completes the proof. 


\section{Proof of Proposition 2}

By Proposition 1 and Lemma 2, it holds under (6),

$$
\begin{aligned}
\widetilde{f}_{\nabla^{r} z}(\lambda) & =\lambda^{-2 d} p^{2 d-1} f_{e}\left(\frac{\lambda}{p}\right)\left[T_{0}(\lambda)\right]^{r+1}+\widetilde{R}_{r}(\lambda) \\
& =\lambda^{-2 d}\left[f_{e}\left(\frac{\lambda}{p}\right)\left(p^{2 d+2 r+1}+O\left(\lambda^{2}\right)\right)+\lambda^{2 d} \widetilde{R}_{r}(\lambda)\right] \\
& \widetilde{R}_{r}(\lambda)=\frac{1}{p} \sum_{j=1}^{p-1} f_{y}\left(\frac{\lambda+2 \pi j}{p}\right)\left[T_{j}(\lambda)\right]^{r+1}
\end{aligned}
$$

With Assumption 3 it follows from Lemma 2 that $\widetilde{R}_{r}(\lambda)=O\left(\lambda^{2 r+2}\right)$ and $\widetilde{R}_{r}^{\prime}(\lambda)=O\left(\lambda^{2 r+1}\right)$, as required. This completes the proof.

\section{Proof of Proposition 3}

Lemma 1 yields under (6)

$$
\dot{f}_{y}(\lambda)=\frac{1}{p}\left[\left(\frac{\lambda}{p}\right)^{-2 d} f_{e}\left(\frac{\lambda}{p}\right)+\sum_{j=1}^{p-1} f_{y}\left(\frac{\lambda+2 \pi j}{p}\right)\right]
$$

such that

$$
\dot{\varphi}_{y}(\lambda)=p^{2 d-1} f_{e}\left(\frac{\lambda}{p}\right)+\frac{\lambda^{2 d}}{p} \sum_{j=1}^{p-1} f_{y}\left(\frac{\lambda+2 \pi j}{p}\right),
$$

which defines $\dot{R}_{y}$ with

$$
0<\varphi_{1}=p^{-1} \sum_{j=1}^{p-1} f_{y}\left(\frac{2 \pi j}{p}\right)<\infty
$$

With Assumption 3 one obtains the required rates for $\dot{R}_{y}$ and $\dot{R}_{y}^{\prime}$, which completes the proof. 


\section{References}

Abadir, K. M., W. Distaso, and L. Giraitis (2007). Nonstationarity-extended local whittle estimation. Journal of Econometrics 141, 1353-1384.

Ait-Sahalia, Y., P. A. Mykland, and P. A. Zhang (2005). How often to sample a continuous-time process in the presence of market microstructure noise. Review of Financial Studies 18, 351-416.

Andrews, D. W. K. and P. Guggenberger (2003). A bias-reduced logperiodogram regression estimator for the long-memory parameter. Econometrica 71, 675-712.

Andrews, D. W. K. and Y. Sun (2004). Adaptive local polynomial Whittle estimation of long-range dependence. Econometrica 72, 569-614.

Baillie, R. T., C.-F. Chung, and M. A. Tieslau (1996). Analysing inflation by the fractionally integrated ARFIMA-GARCH model. Journal of Applied Econometrics 11, 23-40.

Bollerslev, T. and H. O. Mikkelsen (1996). Modeling and pricing long memory in stock market volatility. Journal of Econometrics 73, 151-184.

Breidt, F. J., N. Crato, and P. de Lima (1998). The detection and estimation of long memory in stochastic volatility. Journal of Econometrics 83, 325348.

Brewer, K. R. W. (1973). Some consequences of temporal aggregation and systematic sampling for ARMA and ARMAX models. Journal of Econometrics 1, 133-154.

Chambers, M. J. (1996). The estimation of continuous parameter longmemory time series models. Econometric Theory 12, 374-390.

Chambers, M. J. (1998). Long memory and aggregation in macroeconomic time series. International Economic Review 39, 1053-1072. 
Chambers, M. J. (2004). Testing for unit roots with flow data and varying sampling frequency. Journal of Econometrics 119, 1-18.

Christiano, L. J., M. Eichenbaum, and D. Marshall (1991). The permanent income hypothesis revisited. Econometrica 59, 397-423.

Dalla, V. and J. Hidalgo (2005). A parametric bootstrap test for cycles. Journal of Econometrics 129, 219-261.

Diebold, F. X. and G. D. Rudebusch (1989). Long memory and persistence in aggregate output. Journal of Monetary Economics 24, 189-209.

Drost, F. C. (1994). Temporal aggregation of time-series. In J. Kaehler and P. Kugler (Eds.), Econometric Analysis of Financial Markets, pp. 11-21. Heidelberg: Physica.

Drost, F. C. and T. E. Nijman (1993). Temporal aggregation of GARCH processes. Econometrica 61, 909-927.

Geweke, J. and S. Porter-Hudak (1983). The estimation and application of long memory time series models. Journal of Time Series Analysis 4, 221-238.

Giraitis, L., J. Hidalgo, and P. M. Robinson (2001). Gaussian estimation of parametric spectral density with unknown pole. The Annals of Statistics 29, 987-1023.

Granger, C. W. J. and L. Siklos (1995). Systematic sampling, temporal aggregation, seasonal adjustment, and cointegration: Theory and evidence. Journal of Econometrics 66, 357-370.

Gray, H. L., N.-F. Zhang, and W. A. Woodward (1989). On generalized fractional processes. Journal of Time Series Analysis 10, 233-257.

Guggenberger, P. and Y. Sun (2006). Bias-reduced log-periodogram and Whittle estimation of the long-memory parameter without variance inflation. Econometric Theory 22, 863-912. 
Hansen, L. P. and T. J. Sargent (1983). The dimensionality of the aliasing problem in models with rational spectral densities. Econometrica 51,377387.

Hassler, U. and J. Wolters (1995). Long memory in inflation rates: International evidence. Journal of Business \& Economic Statistics 13, 37-45.

Hidalgo, J. (2005). Semiparametric estimation for stationary processes whose spectra have an unknown pole. The Annals of Statistics 33, 1843-1889.

Hurvich, C. M. and W. W. Chen (2000). An efficient taper for overdifferenced series. Journal of Time Series Analysis 21, 155-180.

Hurvich, C. M., R. Deo, and J. Brodsky (1998). The mean squared error of Geweke and Porter-Hudak's estimator of the memory parameter of a long-memory time series. Journal of Time Series Analysis 19, 19-46.

Hurvich, C. M., E. Moulines, and P. Soulier (2005). Estimating long memory in volatility. Econometrica 73, 1283-1328.

Hurvich, C. M. and B. Ray (2003). The local Whittle estimator of longmemory stochastic volatility. Journal of Financial Econometrics 1, 445470.

Hwang, S. (2000). The effects of systematic sampling and temporal aggregation on discrete time long memory processes and their finite sample properties. Econometric Theory 16, 347-372.

Iouditsky, A., E. Moulines, and P. Soulier (1999). Adaptive estimation of the fracional differencing coefficient. Bernoulli \%, 699-731.

Lobato, I. N. and P. M. Robinson (1996). Averaged periodogram estimation of long memory. Journal of Econometrics 73, 303-324.

Lütkepohl, H. (1987). Forecasting Aggregated Vector ARMA Processes. Springer. 
Lütkepohl, H. (2009). Forecasting aggregated time series variables: A survey. EUI Working Papers 17.

Man, K. S. and G. C. Tiao (2006). Aggregation effect and forecasting temporal aggregates of long memory process. International Journal of Forecasting 22, 267-281.

Mandelbrot, B. B. and J. W. van Ness (1968). Fractional Brownian motion, fractional noises and applications. SIAM Review 10, 422-437.

Marcellino, M. (1999). Some consequences of temporal aggregation in empirical analysis. Journal of Business $\&$ Economic Statistics 17, 129-136.

Marinucci, D. and P. M. Robinson (1999). Alternative forms of fractional Brownian motion. Journal of Statistical Planning and Inference 80, 111122 .

Mishkin, F. S. (2007). Inflation dynamics. International Finance 10, 317334.

Moulines, E. and P. Soulier (1999). Broadband log-periodogram regression of time series with long-range dependence. The Annals of Statistics 27, 1415-1439.

Palm, F. C. and T. E. Nijman (1984). Missing observations in the dynamic regression model. Econometrica 52, 1415-1435.

Paya, I., A. Duarte, and K. Holden (2007). On the relationship between inflation persistence and temporal aggregation. Journal of Money, Credit and Banking 39, 1521-1531.

Pons, G. (2006). Testing monthly seasonal unit roots with monthly and quarterly information. Journal of Time Series Analysis 27, 191-210.

Priestley, M. B. (1981). Spectral Analysis and Time Series, Vol. 1. Academic Press. 
Robinson, P. M. (1994). Rates of convergence and optimal bandwidth in spectral analysis of processes with long range dependence. Probability Theory and Related Fields 99, 443-473.

Robinson, P. M. (1995a). Gaussian semiparametric estimation of long range dependence. Annals of Statistics 23, 1630-1661.

Robinson, P. M. (1995b). Log-periodogram regression of time series with long range dependence. Annals of Statistics 23, 1048-1072.

Robinson, P. M. (2005). The distance between rival nonstationary fractional processes. Journal of Econometrics 128, 283-399.

Rossana, R. J. and J. J. Seater (1995). Temporal aggregation and economic time series. Journal of Business \& Economic Statistics 13, 441-451.

Shiller, R. J. and P. Perron (1985). Testing the random walk hypothesis. Economics Letters 18, 381-386.

Shimotsu, K. (2010). Exact local Whittle estimation of fractional integration with unknown mean and trend. Econometric Theory 26, 501-540.

Shimotsu, K. and P. C. B. Phillips (2005). Exact local Whittle estimation of fractional integration. The Annals of Statistics 33, 1890-1933.

Silvestrini, A. and D. Veredas (2008). Temporal aggregation of univariate and multivariate time series models: A survey. Journal of Economic Surveys 22, 458-497.

Soulier, P. (2001). Moment bounds and central limit theorem for functions of Gaussian vectors. Statistics \& Probability Letters 54, 193-203.

Souza, L. R. (2003). A note on Chambers's "long memory and aggregation in macroeconomic time series". EPGE Ensaios Econômicos Working Paper 503. 
Souza, L. R. (2005). A note on Chambers's "long memory and aggregation in macroeconomic time series". International Economic Review 46, 10591062.

Souza, L. R. (2007). Temporal aggregation and bandwidth selection in estimating long memory. Journal of Time Series Analysis 28, 701-722.

Souza, L. R. (2008). Why aggregate long memory time series? Econometric Reviews 27, 298-316.

Souza, L. R. and J. Smith (2002). Bias in the memory parameter for different sampling rates. International Journal of Forecasting 18, 299-313.

Stock, J. H. and M. W. Watson (2007). Why has U.S. inflation become harder to forecast? Journal of Money, Credit and Banking 39, 3-33.

Stram, D. O. and W. W. S. Wei (1986). Temporal aggregation in the ARIMA process. Journal of Time Series Analysis 7, 279-292.

Sun, Y. and P. C. B. Phillips (2003). Nonlinear log-periodogram regression for perturbed fractional processes. Journal of Econometrics 115, 355-389.

Teles, P., W. W. S. Wei, and N. Crato (1999). The use of aggregate time series in testing for long memory. Bulletin of the International Statistical Institute, 52nd Session 3, 341-342.

Tsai, H. and K. S. Chan (2005a). Temporal aggregation of stationary and nonstationary continuous-time processes. Scandinavian Journal of Statistics 32, 583-597.

Tsai, H. and K. S. Chan (2005b). Temporal aggregation of stationary and nonstationary discrete-time processes. Journal of Time Series Analysis 26, $613-624$.

Tschernig, R. (1995). Long memory in foreign exchange rates revisited. Journal of International Financial Markets, Institutions \& Money 5, 53-78. 
Velasco, C. (1999a). Gaussian semiparametric estimation of non-stationary time series. Journal of Time Series Analysis 20, 87-127.

Velasco, C. (1999b). Non-stationary log-periodogram regression. Journal of Econometrics 91, 325-372.

Wei, W. W. S. (1981). Effect of systematic sampling on ARIMA models. Communications in Statistics - Theory \& Methods 23(A10), 2389-2398.

Wei, W. W. S. (1990). Time Series Analysis: Univariate and Multivariate Methods. Addison-Wesley.

Weiss, A. A. (1984). Systematic sampling and temporal aggragation in time series models. Journal of Econometrics 26, 271-282.

Woodward, W. A., Q. C. Cheng, and H. L. Gray (1998). A k-factor GARMA long-memory model. Journal of Time Series Analysis 19, 485-504. 